\title{
Traduire
}

Une autre perspective sur r tr traduction

Revue française de la traduction

$226 \mid 2012$

Face au miroir

\section{Le nœud gordien}

\section{Françoise Wirth}

\section{(2) OpenEdition}

Journals

Édition électronique

URL : http://journals.openedition.org/traduire/156

DOI : 10.4000/traduire.156

ISSN : 2272-9992

\section{Éditeur}

Société française des traducteurs

\section{Édition imprimée}

Date de publication : 1 janvier 2012

Pagination : 82-86

ISBN : 039-773X

ISSN : 0395-773X

\section{Référence électronique}

Françoise Wirth, «Le nœud gordien », Traduire [En ligne], 226 | 2012, mis en ligne le 03 février 2014, consulté le 11 octobre 2020. URL : http://journals.openedition.org/traduire/156 ; DOI : https://doi.org/ 10.4000/traduire.156

Ce document a été généré automatiquement le 11 octobre 2020 


\title{
Le nœud gordien
}

\author{
Françoise Wirth
}

1 Vous connaissez sûrement Bernhard Schlink, peut-être même sans le savoir. Juriste, il a exercé la profession de juge et celle de professeur de droit. C'est aussi un auteur qui a acquis une notoriété certaine, y compris hors des frontières de son pays natal, l'Allemagne. Il a, en effet, écrit le roman à succès Der Vorleser qui a été traduit dans de nombreuses langues (en français sous le joli titre Le liseur) ${ }^{1}$. Vous ne voyez toujours pas? Mais oui, bien sûr, le titre anglais de ce roman est The Reader et il en a été tiré un film en 2008 sous ce même titre (scénario de David Hare, réalisation de Stephen Daldry, avec Kate Winslet notamment). Ce film a donné lieu à controverse et j'aurais sans doute pu en discuter ici. Sauf que ce n'est pas du tout mon propos. Je voulais vous parler de Bernard Schlink pour une toute autre raison.

2 Il y a quelques années, appâté par la réputation de l'auteur et la première phrase alléchante du commentaire de quatrième de couverture ( Traducteur est aussi un métier où l'on risque quotidiennement sa peau... »), un ami m'a offert un autre de ses romans paru dans la collection Folio policier, Le nœeud gordien. C'est la traduction d'un ouvrage assez nettement antérieur puisque Die gordische Schleife est sorti en $1988^{2}$. La traduction française date, quant à elle, de 2001 et on peut imaginer que le succès du Liseur n'est pas étranger à la décision de publier une version française de ce roman mineur, treize ans après la sortie de l'œuvre originale. En effet, disons-le tout net, il ne s'agit pas d'un excellent livre et si j'ai eu envie d'en parler ce n'est pas pour vous conseiller de le lire mais pour étudier la vision qu'il donne de notre profession.

Vous l'avez donc compris, le personnage principal, le «héros» de ce livre est un traducteur. Il y a donc des auteurs qui pensent qu'un traducteur peut faire un héros, voilà qui est sympathique! Enfin, n'exagérons rien car, dès la seconde page, le lecteur apprend que son héros, Georg Polger a, pour des raisons diverses à dominante sentimentale, quitté un cabinet d'avocats de Karlsruhe pour émigrer vers le sud de la France où il s'est mis à la traduction. Son père ne rate d'ailleurs pas une occasion de lui suggérer de chercher un emploi géographiquement plus proche et plus intéressant (eine "bessere" Arbeit ${ }^{3}$ ). Georg a choisi de s'installer dans le village de Cucuron, à une trentaine de kilomètres d'Aix-en-Provence (très curieusement ce village du Vaucluse 
est remplacé dans la traduction française par celui de Cucugnan, qui existe bel et bien mais se trouve dans l'Aude alors que le début du roman de Schlink est très nettement ancré dans le pays d'Aix).

4 Dès la première page, on sait que c'est la galère. Georg est couvert de dettes. Il vient de faire l'aller-retour Cucuron-Aix dans sa Peugeot pourrie pour s'entendre dire par le patron d'une agence de traduction qu'il n'avait rien à lui proposer. Vous penserez peutêtre que notre héros, qui est juriste de formation (tiens, comme l'auteur - celui-ci aurait-il fait des traductions, par hasard ?) aurait profité de cet atout pour rechercher des traductions dans ce domaine ? Pas vraiment. En fait...

Il écrivit des lettres à tout le monde, postula pour n'importe quel type de traductions, des traductions littéraires, techniques, proposa à des avocats français ses connaissances en droit allemand et à des journaux allemands des articles sur la Provence ${ }^{4}$.

5 En bref, il est prêt à tout et il est clair que le simple fait d'être de langue allemande apparaît sans discussion comme une qualification suffisante pour chercher à traduire des textes littéraires et techniques tout autant que juridiques. Reconnaissons toutefois que, dans un premier temps, il ne trouve pas grand-chose. La traduction n'est de toute façon pas un "premier» choix. Il cherche non seulement "n'importe quelle traduction » mais aussi n'importe quoi. Il est un temps projectionniste dans un cinéma d'Avignon, a «la tête pleine de sujets de reportages, de projets de récits et de romans policiers $»^{5}$ (tiens, comme l'auteur ?).

6 Le quotidien du traducteur tel qu'il est présenté au début de ce livre n'est pas très reluisant :

Ce qui dominait son esprit, c'était l'angoisse : quand Maurin allait-il le rappeler ? Et, lorsque le téléphone était coupé, une fois encore, il s'interrogeait : quand dois-je l'appeler? Il avait parlé d'après-demain. Mais si, demain, il recevait des travaux et n'arrivait pas à me joindre? Est-ce qu'il les garderait pour moi ou est-ce qu'il les donnerait à quelqu'un d'autre ?

7 Mais voici qu'un jour, instant magique pour tout traducteur débutant, quand il décroche le téléphone, il a au bout du fil une personne disposée à lui donner du travail. C'est un certain M. Bulnakof, de la société « Bulnakof Traductions SARL » qu'il vient de créer à Cadenet, dans la banlieue aixoise.

8 C'est donc plein d'espoir que Georg se rend, le vendredi suivant, à l'agence en question (pas encore d'internet à cette époque), où il est accueilli à bras ouverts :

Vous avez de l'expérience dans le domaine technique, informatique, vous passez de l'anglais au français et du français à l'anglais [...] [rappelons, s'il en est besoin, que notre héros est germanophone, quant à son expérience dans l'informatique, il n'en a pas été question dans les pages précédentes qui résument pour tant son itinéraire].

9 En revanche, certains détails pratiques sont intéressants et sonnent justes : Georg travaillera avec un dictaphone et une secrétaire de l'agence se chargera de dactylographier sa traduction. On assiste à la négociation sur les tarifs. M. Bulnakof a d'entrée annoncé «je ne peux pas payer plus de trente-cinq centimes le mot» mais Georg résiste crânement selon des principes que ne renierait pas la SFT: «malheureusement, je ne peux pas accepter trente-cinq centimes. Vous pouvez y réfléchir et me téléphoner à l'occasion, mais pour le moment, vos conceptions d'une éventuelle collaboration et les miennes ne semblent pas s'harmoniser ${ }^{8}$. Il finit par obtenir quarante-cinq centimes. 
Georg repart donc chez lui très satisfait avec un manuel informatique en anglais à traduire en français. Nous sommes vendredi, fin de matinée, il doit rendre la première moitié du manuel le lundi qui suit et la seconde moitié le mercredi. Il est vrai que l'on ne nous donne pas d'indication de volume. Il s'agit peut-être d'un très petit manuel? Toujours est-il que notre ami germanophone se met vaillamment à l'ouvrage et «le lundi matin, il avait traduit et dicté les deux tiers du manuel ». Il faut dire quand même qu'il «travailla plus qu'il ne l'avait jamais fait. Ni pour préparer ses examens, ni au cours de sa carrière d'avocat » mais « ce n'était pas à cause de l'importance du manuel, ni des difficultés que présentait la traduction en français du langage informatique anglais » (à l'évidence, tout cela est très simple pour notre juriste allemand). D'ailleurs, le samedi soir, il dîne chez un ami et le dimanche matin, il fait une « courte promenade, pensant qu'il valait mieux pour lui ne pas rester assis pour réfléchir à la traduction de l'option HELP »'.

11 Il faut dire que notre héros est probablement un traducteur hors pair. Contrairement à ce que pourraient laisser penser les anecdotes ci-dessus, il est convaincu d'être un véritable professionnel. Voici comment il s'assure un autre client (les Industries aéronautiques toulonnaises) :

Je lui ai balancé des termes techniques de traductions que j'avais faites l'année dernière, jusqu'à ce qu'il comprenne que, pour ce boulot, il lui fallait un professionnel et, que ce professionnel, c'était moi. ${ }^{10}$

Il y a aussi un "avocat de Montélimar, spécialiste de leasing informatique » qui «voulait rester en contact avec [lui] pour des traductions de l'allemand vers le français ».

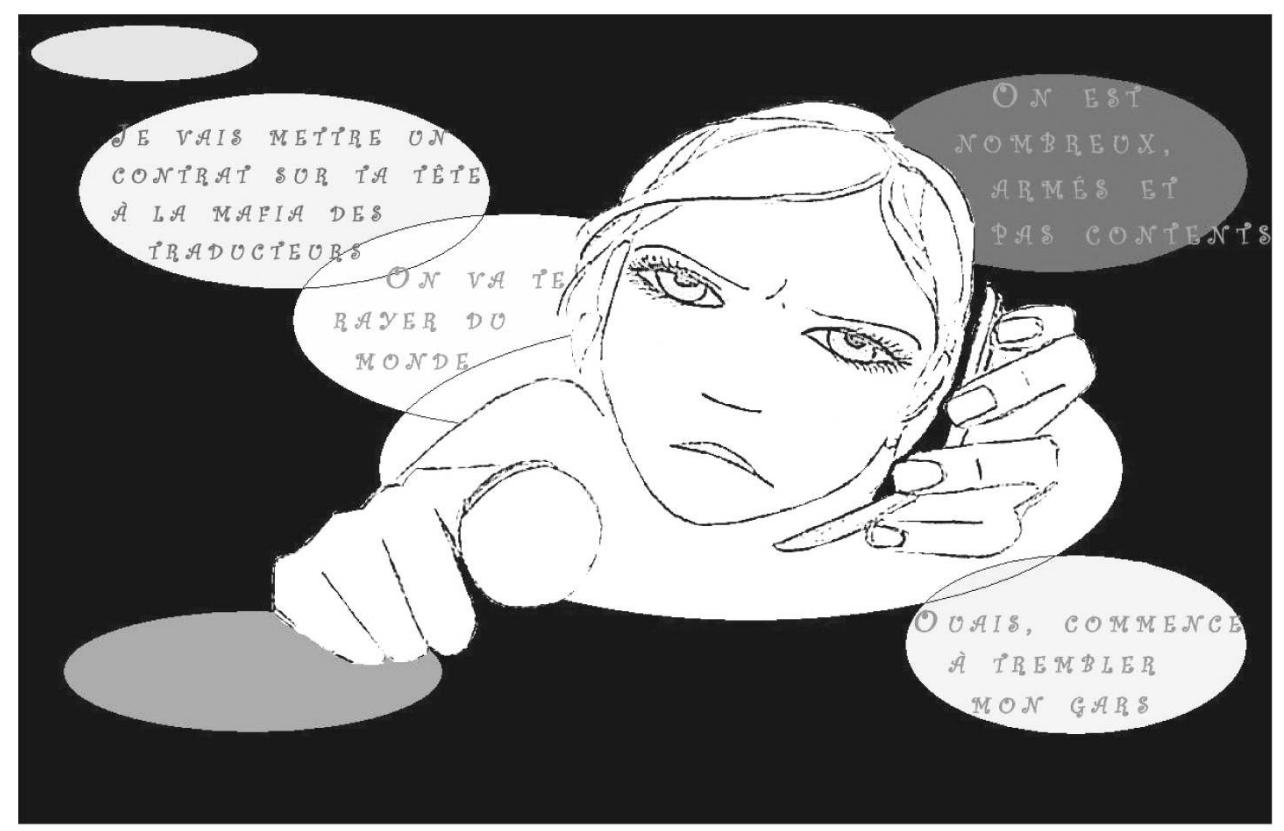

Illustration : Marlène Junius, http://alotoftralala.over-blog.com

Bref, les affaires vont mieux et l'on commence à se dire que, finalement, il a raison : il est possible de vivre de traductions en acceptant de faire n'importe quoi. Il reprend même rapidement les rênes d'une agence de traduction dont le directeur est mort " accidentellement » (car vous vous en souviendrez, il s'agit d'un roman " policier » qui tend d'ailleurs vers le roman d'espionnage). En fait, le contrat pour Bulnakof n'était 
qu'un coup monté et personne ne lira la traduction en français du manuel informatique (c'est peut-être préférable ?) car il ne s'agis- sait que d'un appât. M. Bulnakof est exclusivement intéressé par les plans d'hélicoptère (ultra-secrets) que Georg traduit pour les Industries aéronautiques toulonnaises (là on ne sait pas quelles sont les langues concernées, ce qui compte c'est que notre héros ait les plans chez lui).

Et voici la cerise sur le gâteau. Georg travaille sur les plans d'hélicoptère depuis déjà un certain temps quand il se rend compte que son amie (présentée par Bulnakof) l'espionne et prend les plans en photo la nuit. Du coup : « il passa un certain temps sur les copies et les nouveaux plans pour essayer de comprendre de quoi il s'agissait. Il était question de suspensions, il s'en était rendu compte en traduisant. Mais suspendre quoi et où? ». Notre héros est un super-traducteur mais il ne faut quand même pas lui demander de comprendre ce qu'il traduit... ${ }^{11}$

Le récit évolue ensuite de manière un peu échevelée et je me garderai bien de dévoiler les ressorts de cette histoire complexe et peu crédible. L'importance du métier du personnage principal décroît par la suite. Comme indiqué plus haut, ce métier servait surtout à expliquer la possession par le personnage principal des plans d'un hélicoptère de combat.

Voici, néanmoins, un livre dans lequel le métier de traducteur n'est pas expédié en deux ou trois lignes, comme c'est souvent le cas, mais fait bel et bien l'objet d'un tableau fouillé, s'appuyant sur de nombreux détails pratiques. Peut-être en est-il d'autant plus gênant. Ces détails peuvent, en effet, inciter le lecteur à penser qu'il s'agit d'un portrait documenté et le conforter dans l'idée - déjà trop répandue - qu'un traducteur est quelqu'un qui manie les langues indifféremment les unes des autres, quel que soit le domaine concerné et qui traduit sans vraiment réfléchir à ce qu'il fait, en se contentant de mettre les mots les uns à la suite des autres.

\section{NOTES}

1. Diogenes Verlag, Zürich, 1995. Paris, Folio, trad. Bernard Lortholary, 1999.

2. Le nœud gordien, Bernhard Schlink, trad. Patrick Kermann et Françoise Merle, Gallimard, Folio policier, 2001. Die gordische Schleife, Bernhard Schlink, Diogenes Verlag, Zürich, 1988. Toutes les références ultérieures renvoient à ces deux éditions.

3. p. 8.

4. Er schrieb Briefe an Gott und die Welt, bewarb sich um literarische Übersetzungen, technische Übersetzungen, irgendwelche Übersetzungen, bot französischen Rechtsanwälten seine deutschen Rechtskenntnisse und deutschen Zeitungen Berichte aus der Provence an, p. 19 ; texte allemand p. 13.
5. p. 13.
6. p. 19.
7. p. 29.
8. p. 31 .
9. p. 33.
10. p. 53.
11. p. 89. 
AUTEUR

FRANÇOISE WIRTH

fr.wirth@gmail.com 